\title{
Frequência de incontinência urinária em mulheres praticantes de crossfit: um estudo transversal
}

\author{
Frequency of urinary incontinence in women who practice crossfit: a cross-sectional study
}

Frecuencia de incontinencia urinaria en mujeres que practican crossfit: un estudio transversal Erlon Lopes', Amanda Apolinário², Leila Barbosa ${ }^{3}$, Eduarda Moretti ${ }^{4}$

RESUMO | O objetivo deste estudo foi verificar a frequência de Incontinência Urinaria (IU) em mulheres praticantes de Crossfit. Realizou-se um estudo de corte transversal com mulheres que praticavam Crossfit há, pelo menos, 6 meses ininterruptos, com idade entre 18 a 35 anos e frequência de treino de, no mínimo, três vezes por semana. Foram aplicados questionários de avaliação individual compostos por dados sociodemográficas, antropométricos e esportivos, além do International Consultation on Incontinence Questionnaire - Short Form (ICIQ-UI-SF), para identificar a presença de IU. Também por meio do ICIQ-IU-SF foram identificados a frequência e quantidade de perda de urina e interferência da IU na qualidade de vida (QV) das mulheres incontinentes. Essas mulheres também responderam a um questionário acerca da gravidade da IU. A amostra total do estudo foi de 50 mulheres, das quais 10 (20\%) apresentaram IU. A maioria das mulheres incontinentes apresentou IU de gravidade moderada e de interferência leve na QV. Ademais, observou-se associação entre ter histórico de IU e apresentar IU (RP=5,33; IC95\%=1,41 a 20,10). Diante do exposto, conclui-se que a frequência de IU em mulheres praticantes de Crossfit foi de $20 \%$, sendo a IU, em sua maioria, de gravidade moderada e de leve interferência na QV.

Descritores | Incontinência Urinária; Exercício; Crossfit.

ABSTRACT | The aim of this study was to determine the frequency of urinary incontinence (UI) in women who practice crossfit. This was a cross-sectional study with women aged between 18 and 35 years, who had been practicing crossfit for at least six months without interruption, and at least three times a week. Individual assessment questionnaires were used, composed of sociodemographic, anthropometric and exercise data, as well as the International Consultation on Incontinence Questionnaire - Short Form (ICIQ-UI-SF) to identify the presence of UI. The frequency and amount of urine loss and UI interference in quality of life (QoL) were also identified via ICIQ-IU-SF. These women also responded to a questionnaire about the severity of UI. The total sample of the study was 50 women, of whom 10 (20\%) had UI. Most incontinent women had moderate UI and mild interference in QoL. Moreover, we observed an association between having UI history and presenting UI (PR=5.33, 95\% Cl=1.41 to 20.10). Given the above, we concluded that the frequency of $U I$ in female crossfit athletes was $20 \%$, with UI being mostly of moderate severity and mild interference in QoL.

Keywords | Urinary Incontinence; Exercise; Crossfit.

RESUMEN I El objetivo de este estudio fue evaluar la frecuencia de incontinencia urinaria (IU) en mujeres que practican crossfit. Se realizó un estudio transversal con mujeres que habían practicado crossfit, como mínimo, durante 6 meses ininterrumpidos, con edades entre 18 y 35 años y una frecuencia de entrenamiento de al menos tres veces por semana. Se aplicaron cuestionarios de evaluación individual que constó de datos sociodemográficos, antropométricos y deportivos, además del International Consultation on Incontinence Questionnaire - Short Form (ICIQ-UI-SF) para identificar la presencia de IU. También se identificaron la frecuencia y cantidad de pérdida de orina y la interferencia de la IU en la calidad de vida (CV) de las mujeres con incontinencia utilizando el ICIQ-IU-SF. Esas mujeres también respondieron 
a un cuestionario sobre la gravedad de la IU. La muestra total del estudio se compuso de 50 mujeres, de las cuales 10 (20\%) tenían IU. La mayoría de las mujeres con incontinencia presentaban IU de gravedad moderada e interferencia mínima en la CV. Además, se observó una asociación entre haber presentado antecedentes de IU y tener IU (RP=5,33; IC95\%=1,41 a 20,10). Se concluye que la frecuencia de IU en mujeres que practican crossfit fue del $20 \%$, y que la IU se presentó, en su mayoría, de gravedad moderada y de interferencia mínima en la CV de ellas.

Palabras clave | Incontinencia Urinaria; Ejercicio; Crossfit

\section{INTRODUÇÃO}

A International Urogynecological Association (IUGA) e a International Continence Society (ICS) definem incontinência urinária (IU) como qualquer perda involuntária de urina e a classificam de acordo com o aspecto que induz a perda. Nesse sentido, destacam-se a Incontinência Urinária de Urgência (IUU), que ocorre em momentos de urgência miccional, a Incontinência Urinária de Esforço (IUE), característica de situações com aumento da pressão intra-abdominal, como tosse, espirro e esforços físicos, e Incontinência Urinária Mista (IUM), quando a perda ocorre em ambas as situações ${ }^{1,2}$.

A prevalência de IU em praticantes de atividades físicas é muito variável, dependendo dos tipos de atividades físicas envolvidos ${ }^{3}$. Há estudos que associam a carga de treino, quantidade de tempo (horas/semana) de atividade física e a frequência semanal dos exercícios à perda involuntária de urina ${ }^{4,5}$. Diante disso, mulheres que apresentam IU podem deixar de praticar exercícios devido a incômodos, como os decorrentes de constrangimento. Ademais, entre as atletas, a IU pode comprometer a concentração, o desempenho e a performance nos esportes, além de induzir à restrição hídrica e influenciar no abandono da prática esportiva ${ }^{6-9}$.

No contexto dos exercícios físicos, o Crossfit surge como um novo método de treinamento, o qual envolve a prática de exercícios funcionais e esportivos bastante diversificados, muitas vezes praticados em alta intensidade ${ }^{10,11}$. Os poucos estudos sobre o Crossfit abordam a demanda fisiológica nessa modalidade de exercícios e estão concentrados principalmente nos riscos de lesão e danos musculares ${ }^{12,13}$. Entretanto, deve-se considerar que os exercícios praticados no Crossfit geram um grande aumento da pressão intraabdominal e essa pressão aumentada incide diretamente na musculatura do assoalho pélvico (MAP), podendo influenciar no risco de desenvolver $\mathrm{IU}^{14}$.

Diante do exposto, o objetivo deste estudo foi verificar a frequência de IU em mulheres praticantes de Crossfit. Objetivou-se, ainda, determinar a gravidade da IU e sua interferência na qualidade de vida das mulheres acometidas.

\section{METODOLOGIA}

Trata-se de um estudo de corte transversal, realizado nas academias We Crossfit e Qualimove Crossfit (RecifePE), no período de outubro a novembro de 2018. O projeto segue a Resolução 466/2012 do Conselho Nacional de Saúde e foi aprovado pelo comitê de ética em pesquisa do Centro Universitário Estácio do Recife (CAAE: 95966618.0.0000.5640).

Participaram do estudo mulheres na faixa etária de 18 a 35 anos, menacmes, praticantes de Crossfit há, no mínimo, seis meses ininterruptos e com frequência mínima de treinamento de três vezes por semana. Como critérios de exclusão, foram considerados os seguintes fatores: estar gestante, infecção urinária ativa, afastamento dos treinos por lesões, praticar outro esporte, histórico de doença uroginecológica, cirurgia uroginecológica prévia, doença renal ou neurológica, malformação uroginecológica e câncer pélvico atual ou pregresso.

Os pesquisadores envolvidos foram, inicialmente, treinados quanto aos questionários aplicados. As possíveis participantes foram abordadas antes do início do seu treino ou após finalizá-lo, a fim de verificar os critérios de elegibilidade e o interesse em participar da pesquisa. Caso fossem consideradas elegíveis e concordassem em participar da pesquisa, as voluntárias eram convidadas a ler e assinar o Termo de Consentimento Livre e Esclarecido (TCLE).

Em seguida, as mulheres responderam a uma ficha de avaliação individual, elaborada pelos pesquisadores, contendo informações sociodemográficas, antropométricas e esportivas, além de dados relativos à presença de constipação intestinal e de histórico de IU. Com o objetivo de verificar o autorrelato de IU, as voluntárias responderam, ainda, a sexta questão do questionário International Consultation on Incontinence Questionnaire - Short Form (ICIQ-UI-SF) ${ }^{15}$. Quando a resposta das mulheres indicava presença de IU, era aplicado todo o ICIQ-UI-SF, um questionário traduzido e validado para o português, que visa a identificação do tipo de IU e avaliação da frequência, quantidade e tipo de perda, além da interferência da IU na qualidade de vida ${ }^{15}$. 
Ademais, a fim de avaliar a gravidade da IU, foi também aplicado o Incontinence Severity Index (ISI) ${ }^{16}$, traduzido e validado para a língua portuguesa ${ }^{17}$.

Os dados coletados foram tabulados e analisados no programa Microsoft ${ }^{\circledR}$ Excel, no qual também utilizou-se a calculadora de intervalos de confiança disponibilizada pela PEDro (Physiotherapy Evidence Database) ${ }^{18}$. Uma análise descritiva foi realizada por meio do cálculo de frequências e medidas de tendência central e dispersão, como médias, desvios padrões e intervalos de confiança de 95\% (IC95\%) das variáveis. Além disso, as razões de prevalência (RP) e seus respectivos IC95\% foram calculados a fim de verificar a existência de associação entre presença de IU nas atletas e constipação intestinal, tempo de treino, frequência semanal de treino e histórico de IU nas voluntárias praticantes de Crossfit.

\section{RESULTADOS}

Durante a coleta de dados, 53 mulheres foram consideradas elegíveis, entretanto três mulheres se recusaram a participar do estudo, por falta de tempo $(n=2)$ ou razões pessoais $(n=1)$. Portanto, participaram 50 mulheres praticantes de Crossfit, com média de idade de 28,56 anos. A maioria das mulheres era solteira (76\%), nuligesta (94\%) e nulípara (94\%). A renda familiar média foi de $\mathrm{R} \$ 10.000$ e as mulheres tinham uma média de 17,34 anos de estudo. Quanto aos dados relacionados aos treinos, o tempo médio de prática do Crossfit foi de 16,8 meses, com duração de treino de 60 minutos (92\%) e mediana do número de treinos semanais de 5 (Tabela 1 ).

Tabela 1. Características antropométricas, sociodemográficas, urinárias e esportivas das mulheres praticantes de Crossfit. Pernambuco, Brasil, 2018

\begin{tabular}{|c|c|c|}
\hline Variável & $\mathrm{n}$ & $\%$ \\
\hline Idade (anos) ${ }^{a}$ & \multicolumn{2}{|c|}{$28,56 \pm 4,46$} \\
\hline Peso $(\mathrm{kg})^{\text {a }}$ & \multicolumn{2}{|c|}{$63,11 \pm 8,61$} \\
\hline IMC $\left(\mathrm{kg} / \mathrm{m}^{2}\right)^{\mathrm{a}}$ & \multicolumn{2}{|c|}{$23,40 \pm 2,61$} \\
\hline Renda familiar (R\$) a & \multicolumn{2}{|c|}{$10.006 \pm 5.802,74$} \\
\hline Anos de estudo a & \multicolumn{2}{|c|}{$17,34 \pm 2,34$} \\
\hline \multicolumn{3}{|l|}{ Estado civil } \\
\hline Solteira & 38 & 76 \\
\hline Casada & 12 & 24 \\
\hline Incontinência Urinária & 10 & 20 \\
\hline Noctúria & 20 & 40 \\
\hline Urgência miccional & 4 & 8 \\
\hline Constipação Intestinal & 9 & 18 \\
\hline
\end{tabular}

(continua)
Tabela 1. Continuação

$\begin{array}{lcc}\text { Variável } & \text { n } & \% \\ \text { Praticou outro esporte antes do Crossfit } & 32 & 64 \\ \text { Tempo de prática do Crossfit } & & \\ <12 \text { meses } & 29 & 58 \\ \text { Entre 12 e } 24 \text { meses } & 9 & 18 \\ >24 \text { meses } & 12 & 24\end{array}$

a: Valores expressos em média \pm desvio padrão.

Das 50 voluntárias deste estudo, 10 relataram IU. Assim, a frequência de IU observada foi igual a 20\% (IC95\%: $0,1124$ a 0,3304$)$, sendo a maioria das mulheres (80\%) portadoras de IUE. As demais características urinárias das mulheres incontinentes estão presentes na Tabela 2.

Tabela 2. Características dos sintomas urinários das mulheres praticantes de Crossfit que apresentaram incontinência urinária $(n=10)$. Pernambuco, Brasil, 2018

\begin{tabular}{|c|c|c|}
\hline Variável & $\mathrm{n}$ & $\%$ \\
\hline \multicolumn{3}{|l|}{ Tipos de IU } \\
\hline IU de esforço & 8 & 80 \\
\hline IU de urgência & 1 & 10 \\
\hline IU mista & 1 & 10 \\
\hline \multicolumn{3}{|l|}{ Frequência da perda de urina } \\
\hline 1 vezes por semana ou menos & 6 & 60 \\
\hline 2 ou 3 vezes por semana & 3 & 30 \\
\hline Diversas vezes por dia & 1 & 10 \\
\hline \multicolumn{3}{|l|}{ Quantidade de perda de urina } \\
\hline Pequena quantidade & 9 & 90 \\
\hline Moderada quantidade & 1 & 10 \\
\hline \multicolumn{3}{|l|}{$\begin{array}{l}\text { Interferência da IU na qualidade } \\
\text { de vida }\end{array}$} \\
\hline Sem interferência & 4 & 40 \\
\hline Com interferência 2 na EVA & 3 & 30 \\
\hline Com interferência 3 na EVA & 1 & 10 \\
\hline Com interferência 4 na EVA & 1 & 10 \\
\hline Com interferência 5 na EVA & 1 & 10 \\
\hline \multicolumn{3}{|l|}{ Estratégias para manejo da IU } \\
\hline Sim & 7 & 70 \\
\hline Não & 3 & 30 \\
\hline \multicolumn{3}{|c|}{ Relatou voluntariamente a IU a um profissional de saúde } \\
\hline Sim & 4 & 40 \\
\hline Não & 6 & 60 \\
\hline \multicolumn{3}{|l|}{$\begin{array}{l}\text { Questionada sobre a IU por } \\
\text { profissional de saúde }\end{array}$} \\
\hline Sim & 4 & 40 \\
\hline Não & 6 & 40 \\
\hline
\end{tabular}

IU: Incontinência Urinária; EVA: Escala Visual Analógica. 
A RP entre ter histórico de IU e apresentar IU foi de $R P=5,33$ (IC95\%=1,41 a 20,10), enquanto observou-se uma RP de 1,95 entre apresentar IU e ter constipação intestinal (IC95\%: 0,62 a 6,13). Também foi calculada a RP entre o tempo de treino (dicotomizado em: até um ano de prática de Crossfit e mais de um ano) e a presença de IU. Nesse caso, o resultado nos mostrou uma $\mathrm{RP}=1$ (IC95\%: 0,32 a 3,1). A mesma RP foi calculada considerando: até dois anos de treino e mais de dois anos de treino, e foi visto que, nesta amostra, as mulheres que praticam Crossfit há mais de dois anos apresentaram mais IU (RP=2,75; IC95\%: 0,92 a 8,22). Quanto à frequência de treino por semana (dicotomização: $<5$ vezes por semana e ${ }^{3} 5$ vezes por semana), foi visto uma RP de 3,14 (IC95\% de 0,74 a 13,33).

\section{DISCUSSÃO}

A frequência de IU nas mulheres que praticam Crossfit identificada neste estudo foi de $20 \%$, entretanto ao generalizarmos este dado para a população de mulheres praticantes de Crossfit, é possível sugerir que existe 95\% de probabilidade do real valor de frequência de IU nessa população estar entre 11,24\% e 33,04\%. Quanto às mulheres incontinentes, foi visto que a maioria delas apresentou IUE (80\%) e relatou perder urina em pequena quantidade (90\%). Além disso, $60 \%$ das atletas incontinentes apresentavam IU de gravidade moderada, $40 \%$ não relatou impacto na qualidade de vida, $40 \%$ apresentou impacto leve e $20 \%$ impacto moderado.

Corroborando os presentes achados, um estudo de corte transversal realizado com 41 mulheres que praticavam Crossfit (média de idade: 31,5 anos) há, no mínimo seis meses, também constatou uma frequência de $20 \%$ de IU entre as voluntárias ${ }^{19}$. Outro estudo de corte transversal que envolveu uma amostra de 488 mulheres adultas jovens, observou uma prevalência de IU de 24,6\% nas mulheres que realizavam atividades físicas de alto impacto (idade média de 25,68 anos) ${ }^{20}$.

Sabe-se que a perda involuntária de urina pode interferir na qualidade de vida das mulheres que são acometidas ${ }^{21}$. Entretanto, no presente estudo, apesar de as praticantes apresentarem uma IU de gravidade moderada, foi constatado que a IU não tinha impacto ou interferia de maneira leve na qualidade de vida da maioria das praticantes de Crossfit. Acredita-se que esse resultado se justifique pelo fato da maioria das mulheres (90\%) perderem urina em pequena quantidade. Em consonância com o presente estudo, um estudo que envolveu mulheres praticantes de exercícios físicos de alto impacto também identificou, por meio do ICIQ-UI-SF, uma leve interferência da IU na qualidade de vida das atletas acometidas ${ }^{22}$.

Um dos fatores muitas vezes associado à IU é a constipação intestinal. Em julho de 2014 um estudo estabeleceu os seguintes fatores de risco para IUE: mulheres com dois ou mais filhos, que apresentavam tosse crônica, diabetes mellitus e constipação. Nesse estudo, as mulheres constipadas apresentaram 2,8 mais IU que as mulheres não constipadas (RP=2,8; IC95\%: 1,2 a 6,8) ${ }^{23}$. No presente estudo, foi visto que as mulheres que praticam Crossfit e têm constipação intestinal apresentaram 95\% mais IU do que as mulheres que praticam Crossfit e não têm constipação intestinal ( $R P=1,95$; IC95\%: 0,62 a 6,13). Entretanto, o resultado ainda é impreciso para afirmar a existência dessa associação na população de mulheres praticantes de Crossfit.

Também foi calculada a RP entre o tempo de treino e a presença de IU, porém não foi identificada associação entre o tempo de treino comparando as mulheres que treinam há até um ano e as que praticam Crossfit há mais de um ano e a presença de IU (RP=1; IC95\%: 0,32 a 3,1). Diante disso, foi considerado que talvez um ano não seja tempo suficiente para o aparecimento da IU em mulheres praticantes de Crossfit. Outra RP foi, então, calculada considerando um tempo de prática do Crossfit de até dois anos e mais de dois anos de treino, e foi visto que, no presente estudo, as mulheres que praticam Crossfit há dois anos ou mais apresentaram 2,75 vezes mais IU do que as mulheres que praticam Crossfit há menos de dois anos ( $R P=2,75$; IC95\%: 0,92 a 8,22). Porém, não é possível indicar que essa associação existe em mulheres praticantes de Crossfit pois o IC $95 \%$ engloba a nulidade.

$\mathrm{O}$ estudo que investigou a presença de IU em mulheres atletas de esportes de alto impacto identificou uma relação entre o tempo de prática do esporte e a presença de IU. Porém essa relação só foi observada quando o tempo de prática foi dicotomizado em: menor ou igual a oito anos e maior que oito anos (OR=2,7; IC95\%: 1,4 a 4,98) ${ }^{24}$. A amostra do presente estudo foi composta essencialmente por mulheres que praticavam Crossfit há menos de dois anos. Apenas uma pequena parcela da amostra praticava Crossfit há dois anos ou mais (24\%) e o tempo de prática dessas voluntárias não ultrapassou cinco anos. Assim, a ausência de resultados com significância estatística pode ter sido influenciada também pelo tempo de prática do esporte que a amostra apresentava.

Ainda relacionando a IU com o volume de treino do Crossfit, foi calculada a RP entre a frequência de treino semanal e a presença de IU. O resultado da RP foi de 3,14 (IC95\% de 0,74 a 13,33), indicando que, no presente 
estudo, as mulheres que praticam Crossfit e possuem uma frequência de treino semanal igual ou maior a cinco treinos apresentaram 3,14 vezes mais IU do que as mulheres que praticam Crossfit e possuem uma frequência semanal menor que cinco. Entretanto, o IC95\% obtido é impreciso e toca a nulidade, não sendo possível afirmar que essa associação existe na população em questão. Calculou-se, também, a RP entre ter histórico de IU e apresentar IU e foi visto que, na amostra, as mulheres que praticam Crossfit e têm histórico de IU apresentaram 5,33 mais IU do que as mulheres que praticam Crossfit e não têm histórico de IU (RP=5,33; IC95\%=1,41 a 20,10).

Um aspecto relevante a ser abordado é que 6 (60\%) voluntárias que apresentaram IU afirmaram nunca terem sido questionadas por profissionais de saúde, nem relataram espontaneamente a um profissional de saúde os sintomas urinários. Isso pode ser explicado pelo fato de as mulheres pensarem que é algo comum, de fácil resolução, ou por terem vergonha da condição. Entretanto, deve-se ter bastante atenção pois muitas mulheres com IU podem se sentir constrangidas em atividades sociais e práticas esportivas, além de menos atraídas para a relação sexual, o que pode influenciar no aparecimento de sintomas depressivos, diminuição da autoestima e aumento da ansiedade ${ }^{23}$. Assim, sugere-se que os profissionais que acompanham atletas de Crossfit sejam orientados a prestar uma melhor assistência quanto aos sintomas urinários que as atletas possam apresentar.

Por fim, é importante ressaltar duas limitações importantes do presente estudo. Primeiramente, a não realização do cálculo amostral, que possivelmente contribuiu para a imprecisão encontrada nos resultados obtidos. Diante da característica dos IC95\%, acredita-se que um aumento da amostra pode favorecer o aparecimento da associação entre as variáveis estudadas. Outro aspecto que pode ter interferido nos resultados foi o fato de que a maioria da amostra do presente estudo praticava Crossfit há um ano ou menos, tempo que talvez seja insuficiente para observar a presença de IU entre as mulheres da amostra e suas associações.

\section{CONCLUSÃO}

Uma frequência de IU de 20\% foi observada nas mulheres praticantes de Crossfit. A IUE foi o tipo mais comum e a maioria das mulheres apresentavam uma IU de gravidade moderada e com leve impacto na qualidade de vida. Os relatos das mulheres incontinentes mostraram uma predominância de ocorrência dos episódios de perda urinária de uma vez por semana ou menos e em pequena quantidade. Ademais, foi vista associação estatisticamente significante entre ter histórico de IU e apresentar IU.

\section{REFERÊNCIAS}

1. Haylen BT, Ridder D, Freeman RM, Swift SE, Berghmans B, Lee J, et al. An International Urogynecological Association (IUGA)/International Continence Society (ICS) joint report on the terminology for female pelvic floor dysfunction. Int Urogynecol J. 2010;29(1):5-26. doi: 10.1007/s00192-009-0976-9

2. Leroy LS, Lopes MHBM, Shimo AKK. A incontinência urinária em mulheres e os aspectos raciais: uma revisão de literatura. Texto Contexto Enferm. 2012;21(3):692-701. doi: 10.1590/ S0104-07072012000300026

3. Eliasson K, Larsson T, Mattsson E. Prevalence of stress incontinence in nulliparous elite trampolinists. Scand J Med Sci Sports. 2002;12(2):106-10. doi: 10.1034/j.1600-0838.2002.120207.x

4. Da Roza T, Brandao S, Mascarenhas T, Jorge RN, Duarte JA. Urinary incontinence and levels of regular physical exercise in young women. Int J Sports Med. 2015;36(9):776-80. doi: 10.1055/s-0034-1398625

5. Hannestad YS, Rortveit G, Sandvik H, Hunskaar S. A community-based epidemiological survey of female urinary incontinence: epidemiology of incontinence in the County of Nord-Trondelag. J Clin Epidemiol. 2000;53(11):1150-7. doi: 10.1016/s0895-4356(00)00232-8

6. Eliasson K, Edner A, Mattsson E. Urinary incontinence in very young and mostly nulliparous women with a history of organized high-impact trampoline training: occurrence and risk factors. Int Urogynecol J Pelvic Floor Dysfunct. 2008;19(5):687-96. doi: 10.1007/s00192-007-0508-4

7. Oliveira D, Marques A, Sá-Couto P. Prevalence and impact of urinary incontinence among female athletes. Int J Gynaecol Obstet. 2011;114(1):60-3. doi: 10.1016/j.ijgo.2011.02.004

8. Brawn WJ, Miller YD. Too wet to exercise? Leaking urine as a barrier to physical activity in women. J Sci Med Sport. 2001;4(4):373-8. doi: 10.1016/S1440-2440(01)80046-3

9. Tibana RA, Almeida LA, Prestes J. Crossfit riscos ou benefícios? $\mathrm{O}$ que sabemos até o momento? Rev Bras Cienc Mov. 2015;23(1):182-5. doi: 10.18511/0103-1716/rbcm.v23n1p182-185

10. Tibana RA, Farias DL, Nascimento DC, Silva-Grigoletto ME, Prestes J. Relação da força muscular com o desempenho no levantamento olímpico em praticantes de Crossfit. Rev Andal Med Deport. 2018;11(1):84-8. doi: 10.1016/j.ramd.2015.11.005

11. Tibana RA, Souza NMF, Prestes J. Crossfit: uma análise baseada em evidências. Rev Bras Prescr Fisiol Exerc. 2017;70(1):888-91. Available from: http://www.rbpfex.com.br/index.php/rbpfex/ article/view/1311

12. Joonndeph SA, Joondeph BC. Retinal detachment due to crossfit training injury. Case Rep Ophthalmol Med. 2013;7(3):189-93. doi: 10.1155/2013/189837

13. Da Rosa T, Brandão S, Mascarenhas T, Jorge R, Duarte JA. Urinary incontinence and levels of regular physical exercise 
in young women. Int J Sports Med. 2015;36(9):776-80. doi: 10.1055/s-0034-1398625

14. Bø K, Finckenhagen HB. Vaginal palpation of pelvic floor muscle strength: inter-test reproducibility and comparison between palpation and vaginal squeeze pressure. Acta Obstet Gynecol Scand. 2001;80(10) 883-7. doi: 10.1034/j.1600-0412.2001.801003.x

15. Tamanini JTN, Dambros M, D’Ancona CAL, Palma PCR, Netto Jr. NR. Validação para o português do "International Consultation on Incontinence Questionnaire - Short form" (ICIQ-SF). Rev. Saúde Pública. 2004;38(3):438-44. doi: 10.1590/ S0034-89102004000300015

16. Sandvik H, Hunskaar S, Seim A, Hermstad R, Vanvik A, Bratt H. Validation of a severity index in female urinary incontinence and its implementation in an epidemiological survey. J Epidemiol Community Health. 1993;47(6):497-9. doi: 10.1136/jech.47.6.497

17. Pereira VS, Santos JYC, Correia GN, Driusso P. Tradução e validação para a língua portuguesa de um questionário para avaliação da gravidade da incontinência urinária. Rev Bras Ginecol Obstet. 2011;33(4):182-7. doi: 10.1590/ S0100-72032011000400006

18. Physiotherapy Evidence Database. Confidence interval calculator [Internet]. Sydney; 2013 [cited 2020 Nov 5]. Available from: https://www.pedro.org.au/portuguese/downloads/ confidence-interval-calculator/
19. Bogéa M, Gomes P, Dinnucci A, Dutra F, Zaidan P. Incontinência urinária de esforço em mulheres praticantes de Crossfit: Um estudo transversal de prevalência. Int J Develop Res. 2018;8(7):21642-5.

20. Fozzatti C, Riccetto C, Herrmann V, Brancalion MF, Raimondi M, Nascif $\mathrm{CH}$, et al. Prevalence study of stress urinary incontinence in women who perform high-impact exercises. Int Urogynecol J. 2012;23:1687-91. doi: 10.1007/s00192-012-1786-z

21. Henkes DF, Fiori A, Carvalho JAM, Tavares KO, Frare JC. Incontinência urinária: o impacto na vida de mulheres acometidas e o significado do tratamento fisioterapêutico. Semina Cienc Biol Saúde. 2015;36(2):45-56. doi: 10.5433/1679-0367.2015v36n2p45

22. Cardoso AMB, Lima CROP, Ferreira CWS. Prevalence of urinary incontinence in high-impact sports athletes and their association with knowledge, attitude and practice about this dysfunction. Eur J Sport Sci. 2018:18(10):1405-12. doi: 10.1080/17461391.2018.1496146

23. Caetano AS, Tavares MCGCF, Lopes MHBM, Poloni RL. Influência da atividade física na qualidade de vida e auto-imagem de mulheres incontinentes. Rev Bras Med Esporte. 2009;15(2):93-7. doi: 10.1590/S1517-86922009000200002

24. Thyssen HH, Clevin L, Olosen S, Lose G. Urinary incontinence in elite female athletes and dancers. Int Urogynecol J Pelvic Floor Dysfunct. 2002;13(1):15-7. doi: 10.1007/s001920200003 